\title{
Lipoprotein lipase in hypothalamus is a key regulator of body weight gain and glucose homeostasis in mice
}

\author{
Elise Laperrousaz ${ }^{1}$ - Valentine S. Moullé ${ }^{1} \cdot$ Raphaël G. Denis $^{1} \cdot$ Nadim Kassis $^{1}$ • \\ Chloé Berland $^{1,2,3} \cdot$ Benoit Colsch $^{4} \cdot$ Xavier Fioramonti $^{5}$ - Erwann Philippe ${ }^{1}$. \\ Amélie Lacombe $^{1}$ - Charlotte Vanacker ${ }^{6}$. Noémie Butin ${ }^{4}$ - Kimberley D. Bruce ${ }^{7}$. \\ Hong Wang ${ }^{7}$ - Yongping Wang ${ }^{7}$ Yuanqing Gao ${ }^{2,3}$ - Cristina Garcia-Caceres ${ }^{2,3}$. \\ Vincent Prévot $^{5}$ - Matthias H. Tschöp ${ }^{2,3,8}$ • Robert H. Eckel ${ }^{7}$ • Hervé Le Stunff ${ }^{1}$ • \\ Serge Luquet ${ }^{1}$. Christophe Magnan ${ }^{1}$. Céline Cruciani-Guglielmacci ${ }^{1}$
}

Received: 1 December 2016 / Accepted: 16 March 2017/Published online: 29 April 2017

(C) Springer-Verlag Berlin Heidelberg 2017

\begin{abstract}
Aims/hypothesis Regulation of energy balance involves the participation of many factors, including nutrients, among which are circulating lipids, acting as peripheral signals informing the central nervous system of the energy status of the organism. It has been shown that neuronal lipoprotein lipase (LPL) participates in the control of energy balance by hydrolysing lipid particles enriched in triacylglycerols. Here, we tested the hypothesis that LPL in the mediobasal hypothalamus (MBH), a well-known nucleus implicated in the regulation of metabolic homeostasis, could also contribute to the regulation of body weight and glucose homeostasis.
\end{abstract}

Elise Laperrousaz and Valentine S. Moullé contributed equally to this work.

Yongping Wang, who took part in this research, died before this work was published.

Electronic supplementary material The online version of this article (doi:10.1007/s00125-017-4282-7) contains peer-reviewed but unedited supplementary material, which is available to authorised users.

Christophe Magnan

Christophe.magnan@univ-paris-diderot.fr

$\checkmark$ Céline Cruciani-Guglielmacci

cruciani@univ-paris-diderot.fr

1 Unité de Biologie Fonctionnelle et Adaptative, Sorbonne Paris Cité, CNRS UMR 8251, Université Paris Diderot, Bâtiment Buffon, P. O. box 7126, 4, rue Marie-Andrée Lagroua Weill-Halle, 75205 Paris Cedex 13, France

2 Institute for Diabetes and Obesity, Helmholtz Diabetes Center at Helmholtz Zentrum, Munich, Germany

3 German Center for Diabetes Research (DZD), München-Neuherberg, Germany
Methods We injected an adeno-associated virus (AAV) expressing Cre-green fluorescent protein into the MBH of Lpl-floxed mice (and wild-type mice) to specifically decrease LPL activity in the MBH. In parallel, we injected an AAV overexpressing $\mathrm{Lpl}$ into the MBH of wild-type mice. We then studied energy homeostasis and hypothalamic ceramide content.

Results The partial deletion of $L p l$ in the MBH in mice led to an increase in body weight compared with controls $(37.72 \pm 0.7 \mathrm{~g}$ vs $28.46 \pm 0.12, p<0.001)$ associated with a decrease in locomotor activity. These mice developed hyperinsulinaemia and glucose intolerance. This phenotype

4 CEA-Centre d Etude de Saclay, Laboratoire d étude du Métabolisme des Médicaments, Gif-sur-Yvette, France

5 Centre des Sciences du Goût et de l'Alimentation, Unité Mixte de Recherche CNRS, INRA, Université de Bourgogne, Dijon, France

6 Development and Plasticity of the Neuroendocrine Brain, Neurobese International Associated Laboratory, Jean-Pierre Aubert Research Center, Inserm U1172, University of Lille, Lille, France

7 Division of Endocrinology, Metabolism, \& Diabetes, Department of Medicine, University of Colorado, Denver Anschutz Medical Campus, Aurora, CO, USA

8 Division of Metabolic Diseases, Technische Universität München, Munich, Germany 
also displayed reduced expression of Cers 1 in the hypothalamus as well as decreased concentration of several C18 species of ceramides and a 3 -fold decrease in total ceramide intensity. Conversely, overexpression of $\mathrm{Lpl}$ specifically in the MBH induced a decrease in body weight. Conclusions/interpretation Our study shows that LPL in the $\mathrm{MBH}$ is an important regulator of body weight and glucose homeostasis.

Keywords Hypothalamus · Insulin resistance $\cdot$ Lipoprotein lipase $\cdot$ Nutrient utilisation $\cdot$ Obesity $\cdot$ Triacylglycerol

$\begin{array}{ll}\text { Abbreviations } \\ \text { AAV } & \text { Adeno-associated virus } \\ \text { CNS } & \text { Central nervous system } \\ \text { eGFP } & \text { Enhanced green fluorescent protein } \\ \text { HSP-90 } & \text { Heat shock protein } 90 \\ \text { LPL } & \text { Lipoprotein lipase } \\ \text { MBH } & \text { Mediobasal hypothalamus } \\ \text { RER } & \text { Respiratory exchange rate } \\ \text { TG } & \text { Triacylglycerol }\end{array}$

\section{Introduction}

The control of energy balance by the central nervous system (CNS) involves many peripheral signals, such as hormones (leptin, insulin, ghrelin) and nutrients (glucose, fatty acids, amino acids), and impairment of such control may partly contribute to the onset of obesity and/or type 2 diabetes in predisposed individuals [1]. Among the nutrients, it is now well established that lipids, and more specifically NEFA, are involved in the CNS control of energy balance [2]. NEFA-sensitive neurons are present in key brain regions such as the hypothalamus and may contribute to the regulation of food intake and/or insulin secretion and action (e.g. the control of hepatic glucose production) $[3,4]$. Circulating lipids are also found in lipoproteins such as VLDL or chylomicrons, so-called triacylglycerol (TG)-enriched particles. As with other nutrients, daily variations in the levels of TG in the bloodstream can be detected by the CNS [5] and consequently can also participate in the control of energy balance.

It has been shown that several lipases are expressed in the CNS and can locally hydrolyse TG-enriched particles [6]. In 1990, Ben-Zeev et al showed that the TG-hydrolysing enzyme lipoprotein lipase (LPL) was highly expressed in the rat hippocampus [7]. Several subsequent studies suggested that TG-enriched particles could also modulate lipid sensing through their hydrolysis supplying an alternative source of NEFA in the CNS. Indeed, neuron-specific deletion of $L p l$ induced body weight gain in mice, together with a transient hyperphagia [8]. In this model, the uptake of TG-rich lipoprotein fatty acids was reduced in the hypothalamus, suggesting that a lack of NEFA sensing in the CNS may lead to deregulation of energy balance [8]. We also showed that the partial deletion of $L p l$ specifically in the dorsal hippocampus induced weight gain without overeating in both mice and rats [9]. Decreased locomotor activity and energy expenditure and increased parasympathetic nervous activity were demonstrated in this model [9]. Finally, we recently discovered that dietary TG acted on mesolimbic structures to regulate the rewarding and motivational aspects of feeding in mice, while targeted disruption of LPL specifically in the nucleus accumbens increased palatable food preference and food-seeking behaviour, again suggesting that circulating TG is sensed in the brain by an LPL-dependent mechanism [10]. Altogether, these data highlight a role for LPL in the CNS, especially in areas involved in the regulation of body weight. Consistently, the hypothalamus, the main integrative centre of peripheral signals regulating energy homeostasis, expresses high levels of several genes (particularly the gene encoding LPL) involved in lipid handling, $[6,8]$.

The present work tested the hypothesis that LPL in the mediobasal hypothalamus (MBH, defined as ventromedial hypothalamus plus arcuate nucleus [11]) participates in the regulation of systemic energy balance, as up to $70 \%$ of its neurons are fatty acid sensitive $[4,12]$.

\section{Methods}

Experimental protocol The experimental protocol was approved by the institutional animal care and use committee of the Paris Diderot University (CEEA40), under agreement no. CEB 37.

Animals Eight-week-old male $\mathrm{Lpl}$ loxP mice (strain B6.129S4-Lpltm1 Ijg/J; Jackson Laboratory, Bar Harbor, ME, USA) and wild-type littermates were used to generate $\mathrm{MBH}^{\Delta L p l}$ mice and $\mathrm{MBH}^{L p l}$ mice, respectively [13]. Same-age C57BL6/J mice were used to generate $\mathrm{MBH}^{\text {overLpl }}$ mice and controls after random assignment. CNS neuron-specific LPL-depleted (NexLp $\Gamma^{\prime-}$ ) mice were generated by crossing the $L p l$ loxP mice with transgenic mice having the brain-specific expression of Cre recombinase driven by the regulatory sequences of Nex (also known as Neurod6), a gene that encodes a neuronal basic helix-loop-helix (bHLH) protein [14]. Agrp ${ }^{-1-}$ mice [15] (Merck Research Laboratory, San Francisco, CA, USA) were crossed with $\mathrm{NexLpl}^{/-}$mice to generate neuron-specific LPL-depleted mouse lines, $N e x L p I^{/-}$ and $\mathrm{NexLpl}^{-1-} ; \mathrm{Agrp}^{-1-}$, along with wild-type littermate controls. All mice were housed individually in stainless-steel cages in a room maintained at $22 \pm 1{ }^{\circ} \mathrm{C}$ with lights on from 07:00 hours to 19:00 hours. Mice were given a standard 
laboratory diet (proteins 19.4\%; carbohydrates 59.5\%; lipids $4.6 \%$; vitamins and minerals $16.5 \%$ ) and water ad libitum.

Viral production Adeno-associated viruses (AAVs) of the serotype $2 / 9$, known to have a neuronal tropism, were used $[16,17]$. AAV Cre-enhanced green fluorescent protein (eGFP), was used to induce a recombination within the $\mathrm{MBH}$ in $\mathrm{Lpl}$ loxP mice. The plasmid CBA.nls Cre.eGFP expressing the myc-nls-Cre-GFP fusion protein was kindly provided by R. Palmiter (University of Washington, Seattle, WA, USA $)$ and the AAV2/9 $\left(6 \times 10^{11}\right.$ vector genomes $(\mathrm{vg}) / \mathrm{ml}$ and $1.7 \times 10^{8}$ infectious particles $(\mathrm{ip}) / \mu \mathrm{l}$ ) was produced at UMR Inserm 1089 (Nantes, France). AAV mCherry was used to induce genetic overexpression within the $\mathrm{MBH}$. The plasmid CAG-Lpl-P2AmCherry-WRPE, expressing the mCherry protein, was designed for this experiment and AAV $\left(9.08 \times 10^{11} \mathrm{vg} / \mathrm{ml}\right.$ for the control and $8 \times 10^{11} \mathrm{vg} / \mathrm{ml}$ for the $\mathrm{Lpl}$ ) was produced by Virovek (Hayward, CA, USA).

Surgical and stereotactic procedures At 8 weeks of age, mice were anaesthetised with isoflurane and received an i.p. injection of $180 \mu \mathrm{g} / \mathrm{kg}$ buprenorphine hydrochloride (Axiance, Pantin, France) analgesic before being placed on a stereotaxic frame. AAVs were injected bilaterally into the $\mathrm{MBH}$ (stereotactic coordinates are relative to bregma: $\mathrm{x} \pm 0.5 \mathrm{~mm}$; y $1.64 \mathrm{~mm}$; $5.9 \mathrm{~mm}$ ) at a rate of $0.20 \mu \mathrm{l} / \mathrm{min}$ for a total of $0.5 \mu \mathrm{l}$ per side. At the end of surgical procedures mice received an i.p. injection of $50 \mu \mathrm{g} / \mathrm{kg}$ ketoprofen (Mérial, Lyon, France).

Measurement of food intake, body weight and body composition Food intake and body weight were measured weekly between 09:00 hours and 10:00 hours. Body mass composition was analysed using an Echo Medical Systems EchoMRI 100 (EchoMRI, Houston, TX, USA). Body composition was expressed as a percentage of body weight. Food efficiency was calculated as the body weight gain in $\mathrm{mg}$ divided by the energy intake in $\mathrm{kJ}$.

OGTT Mice were fasted overnight before receiving an oral gavage of glucose $(2 \mathrm{~g} / \mathrm{kg})$. Blood glucose was measured directly from tail vein blood at $0,15,30,45,60,90$ and 120 min using a glucometer (Menarini Diagnostics, Rungis, France). Blood samples were taken from the tail vein at 0,15 and $30 \mathrm{~min}$.

IPITT Mice were fasted for $5 \mathrm{~h}$ before receiving an i.p. injection of insulin $(0.5 \mathrm{U} / \mathrm{kg}$; Novo-Nordisk, La Défense, France). Glycaemia was measured at 0, 15, 30, 45, 60, 90 and $120 \mathrm{~min}$.

Indirect calorimetry Briefly, mice were monitored for whole energy expenditure, oxygen consumption and carbon dioxide production, respiratory exchanged rate (RER; $\left.\dot{V} \mathrm{CO}_{2} / \dot{V} \mathrm{O}_{2}\right)$, food intake and spontaneous locomotor activity using metabolic cages (Labmaster; TSE Systems, Bad Homburg, Germany). Activity was recorded using infrared light beam-based locomotion monitoring system (beam breaks $/ \mathrm{h}$ ). Mice were individually housed and acclimated to the chambers for $48 \mathrm{~h}$ before experimental measurements. Data analysis was carried out with Excel XP (Microsoft France, Issy-Les-Moulineaux, France) using extracted raw values of $\dot{V} \mathrm{O}_{2}, \dot{V} \mathrm{CO}_{2}$ (in $\mathrm{ml} / \mathrm{h}$ ) and energy expenditure $(\mathrm{kJ} / \mathrm{h})$. Subsequently, each value was expressed either per total body weight or whole lean tissue mass extracted from the EchoMRI analysis. See ESM Methods for further details.

Tissue collection Hypothalamus, striatum, total hippocampus and adjacent cortex were dissected following the Glowinski and Iversen technique [18] and immediately frozen.

LPL activity assay Heparin-releasable LPL activity was assayed in brain regions using a Roar LPL activity assay kit (RB-LPL; Roar Biomedical). See ESM Methods for further details.

Quantitative real-time PCR Total RNA was isolated from the hypothalamus, dissected at 10 days or 12 weeks, using RNeasy Lipid Tissue mini kit (Qiagen, Courtaboeuf, France). Quantitative real-time PCR was carried out in a LightCycler 480 detection system (Roche, Meylan, France) using the LightCycler FastStart DNA Master plus SYBR Green I kit (Roche). The mRNA transcript level for each gene was normalised against the mean of two reference genes, Rpl19 and Tbp, which we have previously shown to be unaffected by LPL inhibition (see ESM Table 1 for a list of primers).

Measurement of ceramide levels Total lipid species were extracted according to the method of Folch et al [19]. Ceramide lipid species were enriched in the lower phase (organic phase) and analysed using an untargeted lipidomic approach by liquid chromatography coupled with high-resolution MS (LC-HRMS) as described [20]. MS data treatments were performed using the XCMS software (https://metlin.scripps.edu/).

Analytical measurements Blood samples were collected from the tail vein with heparin. NEFA (Wako, Sobioda, France), triacylglycerol, glycerol (Sigma-Aldrich, Lyon, France), insulin (Crystal Chem, Downers Grove, IL, USA) and leptin (Crystal Chem) were measured according to the manufacturer's instructions.

Western blot Western blots were performed as described previously [10] with minor modifications. Briefly, samples 
were treated with lysis buffer in Tissue-Lyser. Proteins were quantified with the Bradford assay and supplemented with Laemmli buffer $+\beta$-mercaptoethanol. After migration on $10 \%$ acrylamide gel, proteins were transferred on a PVDF membrane (BioRad kit) and incubated overnight with primary antibodies against LPL H-53 (1/1000 in 3\% BSA, Santa Cruz Biotechnology, Dallas, TX, USA) or heat shock protein 90 (HSP-90; H-114, 1/1000 in 3\% BSA, Santa Cruz Biotechnology). Secondary antibody used was HRP anti-rabbit from Santa Cruz, 1/5000 in 3\% BSA, for $2 \mathrm{~h}$. See ESM Methods for further details.

Statistical analysis Data are expressed as means \pm SEM. Statistical analysis was performed using Student's $t$ test or two-way ANOVA followed by two-by-two comparisons using Bonferroni's post hoc test (GraphPad Software, La Jolla, CA, USA). Differences were considered significant at $p<0.05$. Data in representative bar graphs for energy studies are expressed as means \pm SEM of five mice per group extracted from the analysis of variance followed by a Bonferroni's post hoc test (Minitab software, State College, PA, USA) with respective groups, time of day and their interaction as factor. Experimenters were blind to outcome assessment (assays) but not for group assignment. No data were excluded from analysis.

\section{Results}

Hypothalamic LPL deficiency causes body weight gain without hyperphagia $\mathrm{Lpl}$-floxed mice were injected bilaterally into the MBH with a Cre-eGFP-expressing AAV infecting mostly the MBH (Fig. 1a, b). LPL activity was decreased by $\approx 30 \%$ in whole hypothalamus of $\mathrm{Lpl}$-floxed mice compared with control mice, referred to as $\mathrm{MBH}^{\Delta L p l}$ mice and $\mathrm{MBH}^{L p l}$ mice, respectively (Fig. 1c). Cortical LPL activity remained unchanged (Fig. 1c). $\mathrm{MBH}^{\triangle L p l}$ mice developed a rapid weight gain shortly after the $\mathrm{AAV}$ injection, their body weight being significantly different from controls after 3 weeks (Fig. 1d). Interestingly, the obesity was not accompanied by hyperphagia (Fig. 1d). Rather, it was due to a rapid increase in food efficiency, which started shortly after injection (Fig. 1f). Increased body weight was associated with an increased fat mass in absolute value (ESM. Fig. 1a), leading to change in body composition (Fig. 1g). Overall, $\mathrm{MBH}^{\triangle L p l}$ mice demonstrated fasting hyperglycaemia, hyperinsulinaemia and hyperleptinaemia at 12 weeks post injection (Table 1).

Hypothalamic LPL deficiency triggers changes in glucose homeostasis We performed an OGTT at 12 weeks after the AAV injection, when obesity was overt. Consistent with the fat mass increase, mice displayed fasting hyperglycaemia and mild glucose intolerance ( $p$ value for LPL effect $=0.033$ ) associated with a greater insulin response to glucose (Fig. $1 \mathrm{~h}, \mathrm{i}$ ). IPITTs also revealed a strong insulin resistance in $\mathrm{MBH}^{\Delta L p l}$ mice (Fig. 1j).

We then focused on day 10 after the AAV injection, as that was when food efficiency was maximal and mice were neither overweight nor had a changed body composition. Despite elevated plasma NEFA levels in $\mathrm{MBH}^{\triangle L p l}$ mice, key metabolic variables were similar at 10 days post injection in both groups (Table 1). Interestingly, $\mathrm{MBH}^{\Delta L p l}$ mice exhibited impaired glucose tolerance ( $p=0.018)$ (Fig. 2a). In addition, the insulin response to glucose was significantly increased 30 min after the glucose challenge, which corresponded to high blood levels in $\mathrm{MBH}^{\Delta L p l}$ mice, without changes in insulin sensitivity (Fig. 2b, c).

Hypothalamic $L p l$ overexpression slows down body weight gain To confirm the role of LPL in the hypothalamus, we injected an AAV overexpressing $L p l$ using the same stereotaxic coordinates (Fig. 3a). We observed a 30\% increase in LPL protein expression specifically in the hypothalamus of $\mathrm{MBH}^{\text {over } L p l}$ mice compared with $\mathrm{MBH}^{L p l}$ mice, validating relevant overexpression (Fig. 3b, c). As expected, $\mathrm{MBH}^{\text {overL} L p l}$ mice presented a lower body weight compared with $\mathrm{MBH}^{L p l}$ mice (Fig. 3d), even though food intake was no different (Fig. 3i). There were also no differences in glucose tolerance (Fig. 3e, f), insulin sensitivity (Fig. 3g) or body composition (Fig. 3h).

Hypothalamic LPL controls locomotor activity and whole body nutrient utilisation To decipher the mechanisms by which obesity occurs, spontaneous locomotor activity and energy metabolism were assessed by indirect calorimetry. Despite a similar body weight at 10 days, $\mathrm{MBH}^{\Delta L p l}$ mice displayed a significant decrease in locomotor activity, especially at night (Fig. 2e, f), meaning that the lower spontaneous activity cannot be attributed to an obese phenotype but could play a causal role in the setting of obesity. Total energy expenditure was lower (although the difference was not statistically significant) at night in $\mathrm{MBH}^{\Delta L p l}$ mice compared with $\mathrm{MBH}^{L p l}$ mice (Fig. $2 \mathrm{~g}$ ) and the RER was significantly higher for $\mathrm{MBH}^{\Delta L p l}$ mice during both night and day (Fig. 2h, i). Higher RER means greater utilisation of whole body glucose instead of lipid, a potential contributor to increased fat storage and obesity [21, 22]. Consistently, fatty acid oxidation was significantly decreased at day 10 post injection (Fig. 2j, k). At 12 weeks, when $\mathrm{MBH}^{\Delta L p l}$ mice were obese (ESM. Fig. 1a, b), the RER tended to be normalised (ESM. Fig. 2e, f).

Hypothalamic $\mathrm{Lpl}$ overexpression did not change locomotor activity or RER (Fig. 3j, k); energy expenditure was increased, although not significantly (Fig. 31). The 
Fig. 1 LPL deficiency in the hypothalamus induces obesity and insulin resistance without hyperphagia at 12 weeks post injection. (a, b) Fluorescence reflecting AAV2/9 Cre-eGFP infection in the MBH. Scale bar, $200 \mu \mathrm{m}$ (a), $50 \mu \mathrm{m}$ (b). (c) LPL activity in brain expressed in

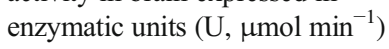
per g of tissue. CTX, cortex; HT, hypothalamus. (d) Time course of body weight. (e) Daily food intake. (f) Food efficiency calculated as the ratio of body weight gain to energy intake. (g) Body composition expressed as percentage of body weight, at 12 weeks. (h, i) Blood glucose levels (h) and plasma insulin levels (i) during the OGTT. (j) Blood glucose levels during the IPITT. Data are means \pm SEM $(n=8-10) . * p<0.05, * * p<0.01$ and $* * * p<0.001 \mathrm{vs} \mathrm{MBH}^{L p l}$ mice. White bars and circles, $\mathrm{MBH}^{L p l}$ mice; black bars and circles, $\mathrm{MBH}^{\Delta L p l}$ mice

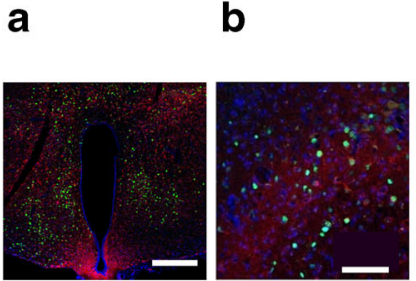

e
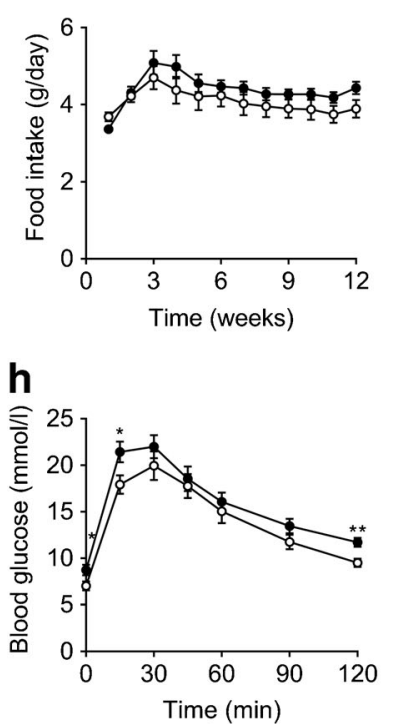

C

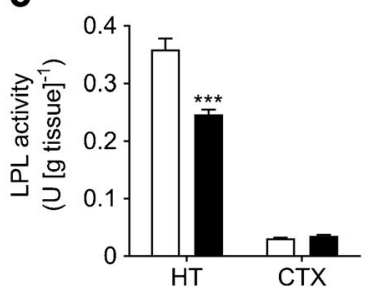

d

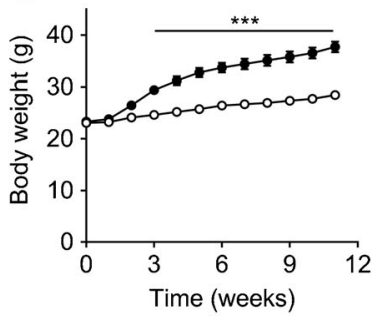

f
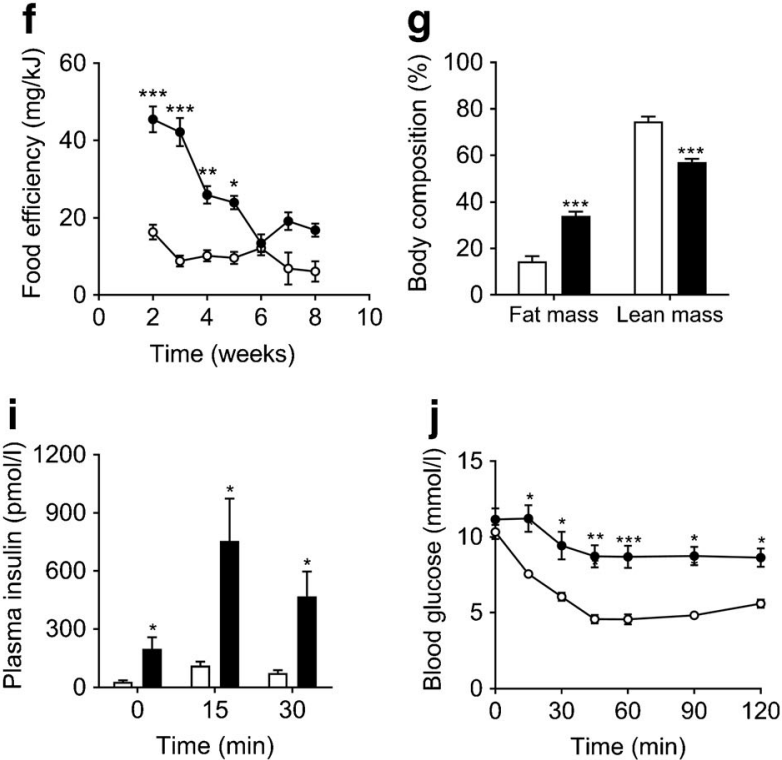

estimated resting energy metabolism was significantly higher during both day and night (Fig. $3 \mathrm{~m}$ ).

\section{Hypothalamic LPL deficiency impairs de novo ceramide} synthesis To determine whether hypothalamic LPL could be involved in ceramide biology, we measured the expression of key enzymes involved in sphingolipid metabolism and performed a lipidomic analysis in $\mathrm{MBH}^{\triangle L p l}$ and $\mathrm{MBH}^{L p l}$ mice. As shown in Table 2, the level of Cers 1 mRNA was downregulated by $33 \%$ at 10 days and by $48 \%$ at 12 weeks in total hypothalamus. Interestingly, Fatpl (also known as Slc27a1; encoding fatty acid transporter 1 , the main transmembrane protein that enhances the uptake of long-chain fatty acids into cells) was downregulated by twofold at 10 days and 12 weeks, likely indicating an adaptive regulation for decreased fatty acid availability following the loss of LPL activity in the hypothalamus. Along with Cers 1 downregulation, $\mathrm{C} 18$ ceramides species (the main by-product
Table 1 Plasma variables in $\mathrm{MBH}^{\Delta L p l}$ and $\mathrm{MBH}^{L p l}$ mice at 10 days and 12 weeks

\begin{tabular}{|c|c|c|c|c|}
\hline \multirow[t]{2}{*}{ Variable } & \multicolumn{2}{|l|}{10 Days } & \multicolumn{2}{|l|}{12 Weeks } \\
\hline & $\mathrm{MBH}^{L p l}$ mice & $\mathrm{MBH}^{\Delta L p l}$ mice & $\mathrm{MBH}^{L p l}$ mice & $\mathrm{MBH}^{\Delta L p l}$ mice \\
\hline Blood glucose (mmol/l) & $9.6 \pm 0.5$ & $9.4 \pm 0.3$ & $8.8 \pm 0.2$ & $11.0 \pm 0.4 * * *$ \\
\hline Leptin $(\mu \mathrm{g} / \mathrm{l})$ & $5.2 \pm 0.5$ & $4.0 \pm 0.8^{*}$ & $5.1 \pm 1.1$ & $20.6 \pm 2.2 *$ \\
\hline NEFA (mmol/l) & $0.41 \pm 0.10$ & $0.86 \pm 0.11^{*}$ & $0.33 \pm 0.10$ & $0.53 \pm 0.06$ \\
\hline $\mathrm{TG}(\mathrm{mmol} / \mathrm{l})$ & $0.64 \pm 0.068$ & $0.69 \pm 0.079$ & $0.54 \pm 0.10$ & $1.92 \pm 0.13 *$ \\
\hline Glycerol (mg/ml) & $0.70 \pm 0.04$ & $0.79 \pm 0.05$ & $0.25 \pm 0.03$ & $0.36 \pm 0.04 *$ \\
\hline Fasting blood glucose (mmol/l) & $6.0 \pm 0.3$ & $6.6 \pm 0.4$ & $6.2 \pm 0.9$ & $8.7 \pm 0.6^{*}$ \\
\hline Fasting insulin (pmol/1) & $56.6 \pm 0.9$ & $61.2 \pm 3.6$ & $33.5 \pm 7.5$ & $198.1 \pm 59.2 *$ \\
\hline Fasting NEFA (mmol/l) & $1.46 \pm 0.18$ & $1.36 \pm 0.06$ & $1.70 \pm 0.11$ & $1.37 \pm 0.06^{*}$ \\
\hline
\end{tabular}

Data are means \pm SEM, $n=8-10$

$* p<0.05$ and $* * * p<0.001$ vs age-matched $\mathrm{MBH}^{L p l}$ mice 


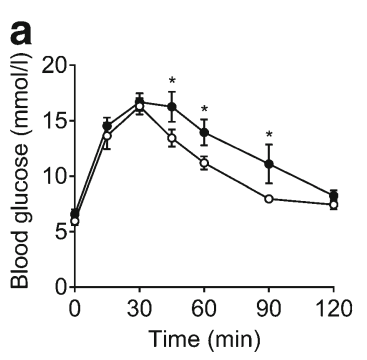

e

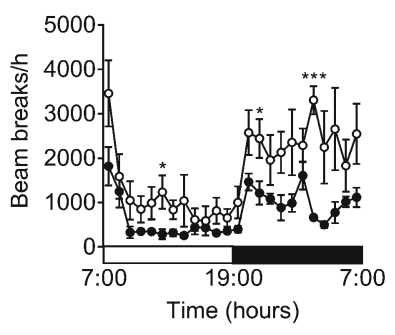

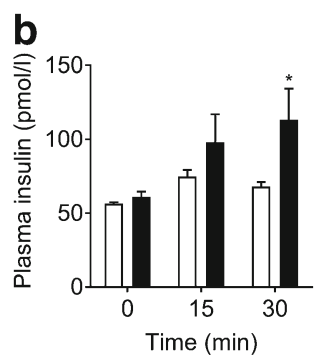

f

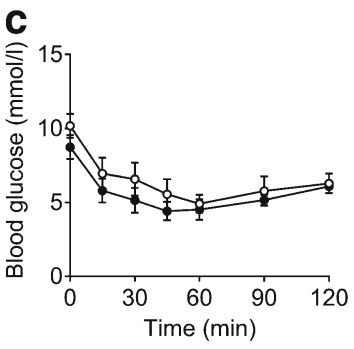

d

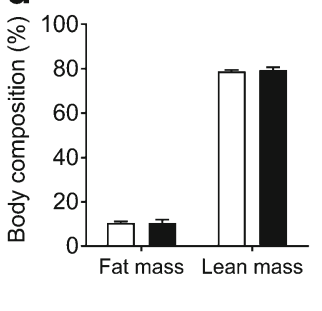

g

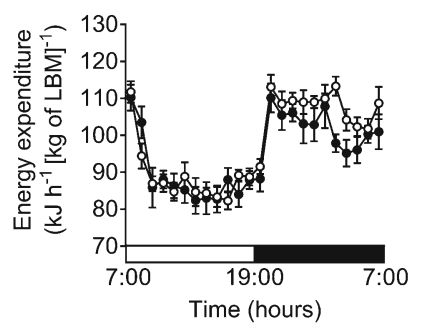

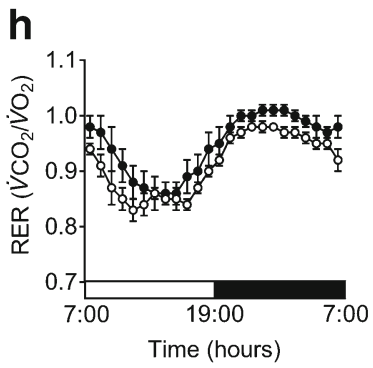

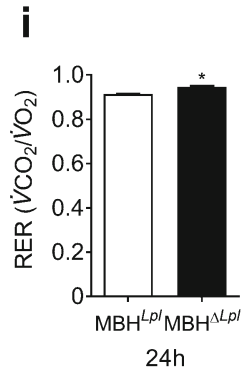

$24 \mathrm{~h}$

Fig. 2 LPL deficiency in the hypothalamus induces early dysfunction of glucose homeostasis and energy metabolism at day 10 post injection. (a, b) Blood glucose levels (a) and plasma insulin levels (b) during the OGTT. (c) Blood glucose levels during the IPITT. (d) Body composition expressed as percentage of body weight. (e, f) Locomotor activity expressed as $24 \mathrm{~h}$ profile representative of $96 \mathrm{~h}$ measurement of $\mathrm{MBH}^{L p l}$ and $\mathrm{MBH}^{\Delta L p l}$ mice (e) and representative bar graph showing the mean extracted from the ANOVA (f). (g) Energy expenditure

of Cers 1 activity [23]) were substantially decreased in the hypothalamus at 10 days (Fig. 4a). Other ceramide species of various chain lengths were also decreased (Fig. 4b-f). These results show that loss of LPL in the MBH leads to a widespread modulation of ceramide levels in other brain areas known to be involved in the control of energy metabolism (ESM Fig. 3). At 12 weeks post injection, the ceramide levels returned to normal (as compared with $\mathrm{MBH}^{L p l}$ mice) in the hypothalamus (Fig. 4a), hippocampus and cortex but remained low in the striatum (ESM Fig. 3). The normalisation is probably due to a compensatory increase in the expression of other ceramide synthase genes (Cers2 and Cers 3 ; cf. Table 2). However, we did not observe any changes in ceramide species levels in the presence of $L p l$ overexpression at 10 days (Fig. 5), although discrete changes in a specific cell compartment could have occurred as it has been demonstrated in vitro [24].

\section{Discussion}

We demonstrated that hypothalamic LPL is an important regulator of metabolic homeostasis in mice. Indeed, hypothalamic LPL deficiency led to severe obesity through an early decrease in locomotor activity and a change in nutrient utilisation in favour of lipid storage. This phenotype was associated with an early impaired glucose homeostasis and a lower hypothalamic ceramide content. Conversely, overexpression of $\mathrm{Lpl}$ specifically in the $\mathrm{MBH}$ induced a decrease in body weight without alteration of locomotor activity or food intake.

LPL is widely expressed in brain areas such as the hippocampus, striatum, hypothalamus, cerebellum and brain stem [25] and has been found in neurons, brain endothelial vessels and glial cells [26]. In our model, we observed a significant $30 \%$ decrease in hypothalamic LPL activity. This 


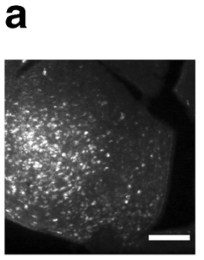

b

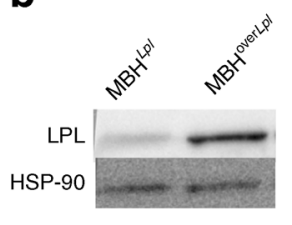

C

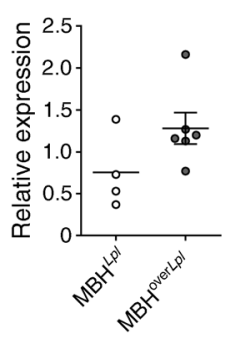

d

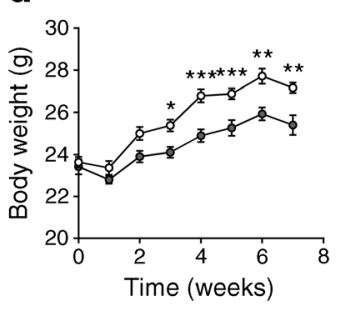

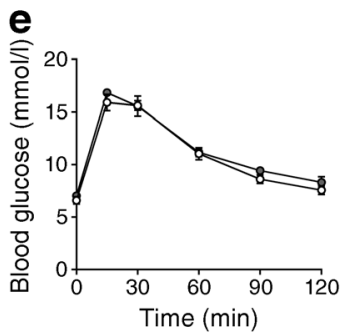

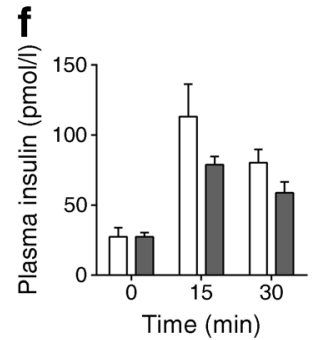

j

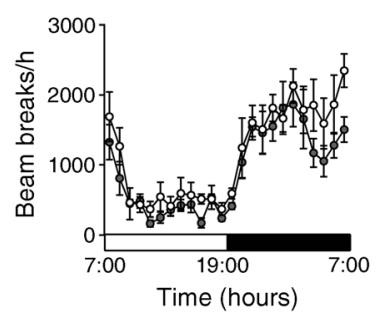

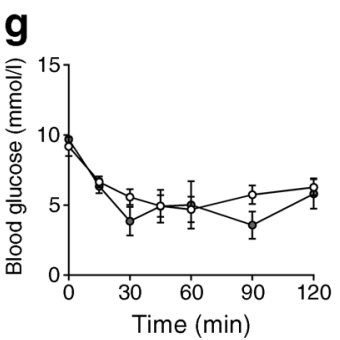

k

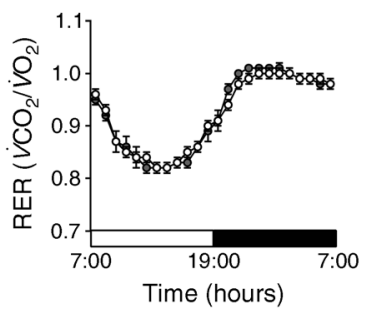

Fig. 3 Overexpression of $\mathrm{Lpl}$ in $\mathrm{MBH}$ decreases body weight gain without decreasing food intake and increases resting energy metabolism at day 10 post injection. (a) Fluorescence reflecting infection with AAV2/9 overexpressing $\mathrm{Lpl}$ in $\mathrm{MBH}$, coupled to mCherry fluorescent protein. Scale bar, $200 \mu \mathrm{m}$. (b, c) Representative western blot (b) and quantification of LPL levels compared with HSP-90 (c). (d-g) Body weight (d), blood glucose levels (e) and plasma insulin levels (f) during the OGTT and blood glucose levels during the IPITT (g). (h) Body

Table 2 Hypothalamic mRNA expression in $\mathrm{MBH}^{\Delta L p l}$ mice at 10 days and 12 weeks

\begin{tabular}{lcc}
\hline Metabolic process/gene & 10 Days & 12 Weeks \\
\hline Ceramide metabolism & & \\
$\quad$ Spt3 & $73 \pm 18$ & $150 \pm 5^{* * *}$ \\
Cers 1 & $67 \pm 6^{*}$ & $53 \pm 4^{* * *}$ \\
Cers2 & $89 \pm 12$ & $138 \pm 12^{*}$ \\
Cers3 & $75 \pm 17$ & $122 \pm 4^{*}$ \\
Lipogenesis & & \\
Srebplc & $81 \pm 13$ & $131 \pm 11^{*}$ \\
Fatp1 & $56 \pm 7^{* *}$ & $51 \pm 4^{* *}$ \\
\hline
\end{tabular}

Data are means \pm SEM and show fold change relative to $\mathrm{MBH}^{L p l}$ mice; $n=3-5$ at 10 days and $n=8-10$ at 12 weeks post injection

Tbp and Rpl19 mRNA levels were used to normalise mRNA expression Spt3 is also known as Sptlc3; Srebp1c is also known as Srebf1 ${ }^{*} p<0.05, * * p<0.01$ and ${ }^{* * *} p<0.001$ vs age-matched $\mathrm{MBH}^{L p l}$ mice
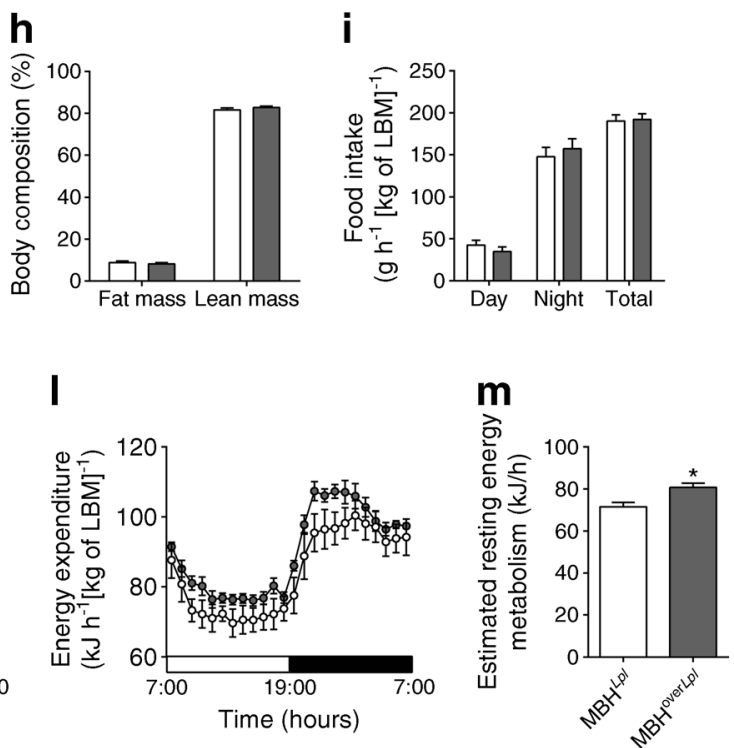

composition expressed as percentage of body weight. (i) Food intake normalised per lean body mass (LBM). (j) Locomotor activity expressed as $24 \mathrm{~h}$ profile representative of $96 \mathrm{~h}$ measurement. (k) RER. (I, m) Energy expenditure normalised per lean body mass (l) and bar graph showing estimated resting energy metabolism mean extracted from the ANOVA $(\mathbf{m})$. Data are means $\pm \operatorname{SEM}(n=5$ or 6$) . * p<0.05, * * p<0.01$ and $* * * p<0.001$ vs $\mathrm{MBH}^{L p l}$ mice. White bars and circles, $\mathrm{MBH}^{L p l}$ mice; dark grey bars and circles, $\mathrm{MBH}^{\text {overLpl }}$ mice

relatively modest reduction may be largely attributed to the neuronal specificity of the knockout since the AAV serotype $2 / 9$ has a neuronal tropism and the chicken $\beta$-actin (CBA) promoter displayed a high neural expression [27]. We had some knowledge that a decrease in neuron-specific LPL expression in different brain areas was associated with alterations in energy homeostasis. In 2011 R. H. Eckel's laboratory showed that mice with neuron-specific loss of LPL (all over the brain, under the Nex promotor [14]) became obese after experiencing weeks of hyperphagia with increased Agrp and Npy expression and then reduced energy expenditure [8]. Moreover, we recently reported that intrahippocampal LPL inhibition in both rats and mice led to increased body weight gain due to decreased locomotor activity and energy expenditure concomitant with high parasympathetic tone, but with no change in food intake [9]. Interestingly we identified de novo ceramide synthesis as a potential molecular mechanism by which altered hippocampal 

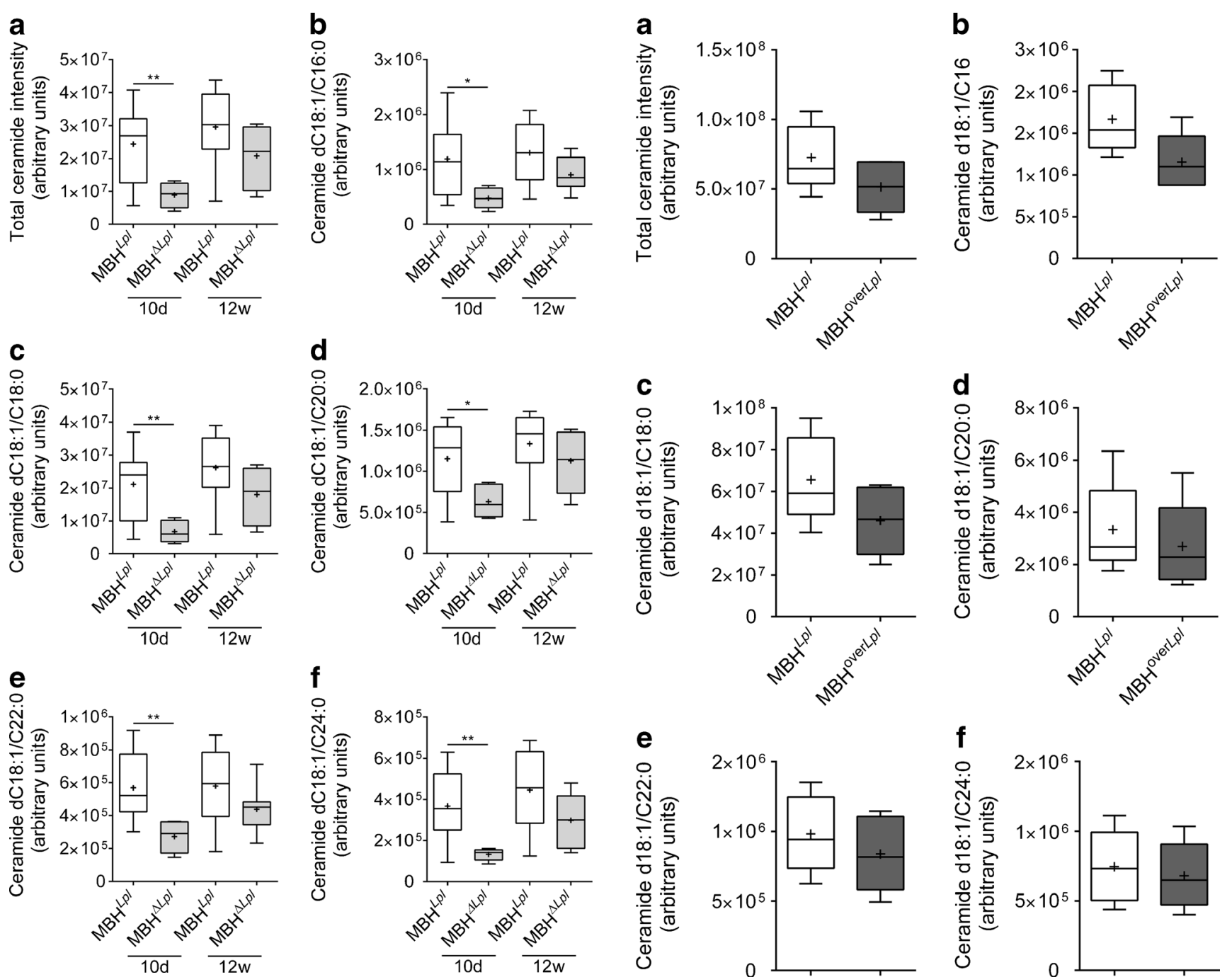

Fig. 4 Hypothalamic ceramide content is decreased in $\mathrm{MBH}^{\Delta L p l}$ mice at 10 days (d) but not 12 weeks (w) post injection. Analyses were performed by MS on the total content (a) and in detail, for ceramide classes $\mathrm{C16}$ (b), $\mathrm{C} 18$ (c), C20 (d), C22 (e) and C24 (f). Chromatographic peak area is shown. The + symbol represents the mean. $* p<0.05$ and $* * p<0.01$ vs $\mathrm{MBH}^{L p l}$ mice $(n \geq 5)$; unpaired Student's $t$ test with Welch's correction. White bars, $\mathrm{MBH}^{L p l}$ mice; light grey bars, $\mathrm{MBH}^{\Delta L p l}$ mice

TG hydrolysis may affect energy balance. This allows us to consider central LPL as being not only a 'tool' for TG hydrolysis and delivery of fatty acids for neuronal fatty acid sensing but also a protein that controls further cellular mechanisms and will ultimately regulate peripheral energy metabolism through the autonomic nervous system.

Here, we targeted MBH, since NEFA-sensitive neurons are highly represented in the MBH [12] and this brain area is key in the regulation of food intake and whole body energy homeostasis through the autonomic nervous system and hypothalamus-pituitary axis. $\mathrm{MBH}^{\triangle L p l}$ mice displayed an increase in food efficiency, especially during the 4 weeks following the injection of the virus, meaning that their capacity to store energy was increased. With a higher RER
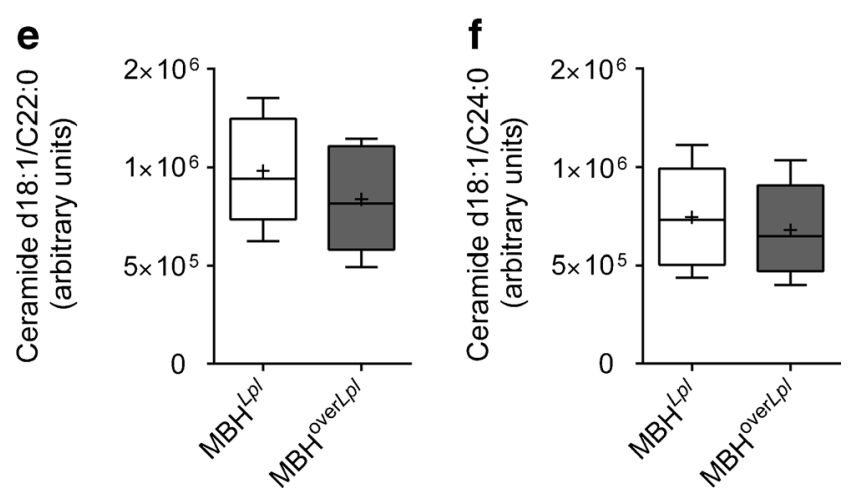

Fig. 5 Hypothalamic ceramide content is not changed in $\mathrm{MBH}^{\text {overL } L l}$ mice at 10 days post injection. Analyses were performed by MS on the total content (a) and in detail, for ceramide classes C16 (b), C18 (c), C20 (d), C22 (e) and C24 (f). Chromatographic peak area is shown. The + symbol represents the mean. $n \geq 5$; unpaired Student's $t$ test with Welch's correction. White bars, $\mathrm{MBH}^{L \overline{p l}}$ mice; dark grey bars, $\mathrm{MBH}^{\mathrm{over} L p l}$ mice

and a lower NEFA oxidation rate, an early difference in the type of nutrients used could be responsible for the development of obesity. Interestingly, food intake remained unchanged and the expression levels of Agrp and Npy were not affected (data not shown), highlighting a metabolic control of body weight rather than a classical modulation of hypothalamic orexigenic/anorexigenic neuropeptides. In addition, we demonstrated here that mice bearing the double knockout for Agrp and $\mathrm{Lpl}$ under the Nex promotor still developed obesity (ESM Fig. 2c), meaning that an increase in Agrp mRNA is not necessary for induction of changes in energy balance and body weight. 
The decrease in locomotor activity observed in $\mathrm{MBH}^{\Delta L p l}$ mice occurred before the onset of obesity and its consequences (hyperglycaemia, hyperinsulinaemia, hyperleptinaemia, insulin resistance). Although we did not observe any significant modifications of energy expenditure, the early decrease in locomotor activity potentially promotes energy storage and the development of obesity. In addition, data obtained at 10 days post injection showed that a defect in glucose tolerance was already present (accompanied by an increased insulin response to glucose) before any weight gain, suggesting that hypothalamic LPL could directly modulate hepatic glucose production, peripheral glucose uptake or insulin secretion through the autonomic nervous system. In humans, even if a link between decreased brain LPL activity and type 2 diabetes has not been documented, it must be pointed out that several studies have demonstrated a decrease in LPL activity in the serum [28]), plasma [29] or adipose tissue $[30,31]$ of individuals with type 2 diabetes. To our knowledge, this is the first time that hypothalamic LPL has been specifically targeted and this study highlights the role of CNS LPL activity in controlling not only body weight but also glucose homeostasis.

We performed a lipidomic analysis in several brain areas, particularly the hypothalamus of $\mathrm{MBH}^{\Delta L p l}$ mice. We showed that the total amount of hypothalamic ceramide species was decreased at 10 days, before the gain in body weight, suggesting that the lack of ceramides could play a causal role in obesity development. At the same time, there was a decrease in Cers 1 gene expression and a transient decrease in hypothalamic ceramides, especially the C18:0 species. Ceramide synthase 1, among the six ceramide synthase isoforms, is mainly involved in the synthesis of C18:0 ceramides, and notably it has been recently linked to a greater insulin sensibility in muscle cells [32]. The overexpression of Cers 1 in myotubes increases the levels of C16:0, C18:0 and total ceramides, ultimately promoting GLUT4 translocation and glycogen synthesis. In the present study, the early decrease in Cers 1 expression in $\mathrm{MBH}^{\Delta L p l}$ mice could mediate a defect in hypothalamic signalling, which has been already shown to be associated with obesity [33]. Consistent with this hypothesis, $\mathrm{MBH}^{\Delta L p l}$ mice had a higher food intake after a $24 \mathrm{~h}$ fast which could indicate a blunted insulin satiety effect (data not shown). In addition, Cers 1 knockout mice have neurodegenerative disorders and impaired locomotor performance. Zhao et al reported that Cers 1 deficiency dramatically affects sphingolipid homeostasis and leads to Purkinje cell loss and lipofuscin accumulation [34]. Moreover, another study reported that Cers1-deficient mice display functional deficits including impaired exploration of novel objects, locomotion and motor coordination [35]. Thus, the observed decrease in Cers 1 expression could play a causal role in the decreased locomotor activity and the development of obesity.
Consistent with this, $L p l$ overexpression in mouse MBH showed a partial 'mirror phenotype'. Weight gain was significantly lower and a higher resting metabolic rate developed in $\mathrm{MBH}^{\text {over } L p l}$ mice compared with $\mathrm{MBH}^{L p l}$ mice. These data confirm the role of LPL as a regulator of body weight gain and its implication in the modulation of energy expenditure. While knockdown of LPL altered MBH ceramide content, overexpression had no consequences on intracellular ceramide content, suggesting that alternative intracellular signals are mobilised in conditions of abundant NEFA entry. Indeed, LPL can provide NEFA to brain cells, which are transformed to reach different fates, such as $\beta$-oxidation, ceramide or endocannabinoid synthesis, therefore exerting metabolic effects through the modulation of autonomic nervous system, as has been already been shown for ceramides [36] and endocannabinoids [37]. A non-exclusive hypothesis would be the presence, in the $\mathrm{MBH}$, of neuronal populations with different ranges of sensitivity for NEFA. Specifically, Le Foll et al showed that oleic acid-excited neurons dose-dependently responded to lower concentrations than oleic acid-inhibited neurons [4]. While LPL knockdown is likely to affect both populations, overexpression will probably have a more pronounced effect in neurons in which NEFA delivery is limited. In turn these populations might differentially control energy expenditure, feeding or glucose metabolism.

To conclude, we showed for the first time that hypothalamic LPL controls body weight and modulates both glucose homeostasis and locomotor activity. Decreased LPL activity in the hypothalamus is accompanied by a transient drop in ceramide content and leads to early dysregulation of glucose homeostasis and nutrient utilisation. Further studies are needed to decipher the mechanisms by which $\mathrm{Lpl}$ overexpression in the hypothalamus could limit body weight gain, and the specific role of $\mathrm{Lpl}$ expressed in astrocytes or microglia remains to be determined. Understanding the functions of LPL in the hypothalamus may potentially provide new therapeutic opportunities.

Acknowledgements We acknowledge the technical platform Functional \& Physiological Exploration Platform (FPE) of the Unit 'Biologie Fonctionnelle et Adaptative' (Université Paris Diderot, Sorbonne Paris Cité, BFA, UMR 8251 CNRS, F-75205 Paris, France) for metabolic analysis. We also thank the animal core facility 'Buffon' of the Université Paris Diderot / Jacques Monod Institute, Paris, for animal husbandry and breeding.

Data availability The data that support the findings of this study are available from the corresponding author upon reasonable request.

Funding This study was supported by a grant from National Research Agency (ANR SVSE 1 2011: 'lipobrain') and a grant from European Foundation for Study of Diabetes (EFSD/Lilly Research Fellowship Programme 2014). 
Duality of interest The authors declare that there is no duality of interest associated with this manuscript.

Contribution statement EL, VSM, CM and CCG performed the study, researched the data, analysed the results and wrote the manuscript. RGD, NK, CB, BC, XF, EP, AL, CV, NB, KDB, HW, YW, YG, CG-C performed experiments, analysed the results and drafted the manuscript. VP, MHT, RHE, HLS, SL and CM analysed the data, drafted and revised the manuscript. EL, VSM, SL, CM and CCG revised the article critically for important intellectual content, and all the authors approved the final version to be published. CCG is the guarantor of this work and, as such, had full access to all the data in the study and takes responsibility for the integrity of the data and the accuracy of the data analysis.

\section{References}

1. Blouet C, Schwartz GJ (2010) Hypothalamic nutrient sensing in the control of energy homeostasis. Behav Brain Res 209:1-12

2. Magnan C, Levin BE, Luquet S (2015) Brain lipid sensing and the neural control of energy balance. Mol Cell Endocrinol 418:3-8

3. Picard A, Moulle VS, Le Foll C et al (2014) Physiological and pathophysiological implications of lipid sensing in the brain. Diabetes Obes Metab 16(Suppl 1):49-55

4. Le Foll C, Irani BG, Magnan C, Dunn-Meynell AA, Levin BE (2009) Characteristics and mechanisms of hypothalamic neuronal fatty acid sensing. Am J Phys Regul Integr Comp Phys 297:R655-R664

5. Ruge T, Hodson L, Cheeseman J et al (2009) Fasted to fed trafficking of fatty acids in human adipose tissue reveals a novel regulatory step for enhanced fat storage. J Clin Endocrinol Metab 94:1781-1788

6. Paradis E, Clavel S, Julien P et al (2004) Lipoprotein lipase and endothelial lipase expression in mouse brain: regional distribution and selective induction following kainic acid-induced lesion and focal cerebral ischemia. Neurobiol Dis 15:312-325

7. Ben-Zeev O, Doolittle MH, Singh N, Chang CH, Schotz MC (1990) Synthesis and regulation of lipoprotein lipase in the hippocampus. J Lipid Res 31:1307-1313

8. Wang H, Astarita G, Taussig MD et al (2011) Deficiency of lipoprotein lipase in neurons modifies the regulation of energy balance and leads to obesity. Cell Metab 13:105-113

9. Picard A, Rouch C, Kassis N et al (2014) Hippocampal lipoprotein lipase regulates energy balance in rodents. Mol Metab 3:167-176

10. Cansell C, Castel J, Denis RG et al (2014) Dietary triglycerides act on mesolimbic structures to regulate the rewarding and motivational aspects of feeding. Mol Psychiatry 19:1095-1105

11. Dunn-Meynell AA, Le Foll C, Johnson MD, Lutz TA, Hayes MR, Levin BE (2016) Endogenous VMH amylin signaling is required for full leptin signaling and protection from diet-induced obesity. Am J Phys Regul Integr Comp Phys 310:R355-R365

12. Levin BE, Magnan C, Dunn-Meynell A, Le Foll C (2011) Metabolic sensing and the brain: who, what, where, and how? Endocrinology 152:2552-2557

13. Augustus A, Yagyu H, Haemmerle G et al (2004) Cardiac-specific knock-out of lipoprotein lipase alters plasma lipoprotein triglyceride metabolism and cardiac gene expression. J Biol Chem 279: 25050-25057

14. Goebbels S, Bormuth I, Bode U, Hermanson O, Schwab MH, Nave KA (2006) Genetic targeting of principal neurons in neocortex and hippocampus of NEX-Cre mice. Genesis 44:611-621

15. Qian S, Chen H, Weingarth D et al (2002) Neither agouti-related protein nor neuropeptide $\mathrm{Y}$ is critically required for the regulation of energy homeostasis in mice. Mol Cell Biol 22:5027-5035
16. Chakrabarty P, Rosario A, Cruz P et al (2013) Capsid serotype and timing of injection determines AAV transduction in the neonatal mice brain. PLoS One 8:e67680

17. Rahim AA, Wong AM, Ahmadi S et al (2012) In utero administration of Ad5 and AAV pseudotypes to the fetal brain leads to efficient, widespread and long-term gene expression. Gene Ther 19: 936-946

18. Glowinski J, Iversen LL (1966) Regional studies of catecholamines in the rat brain. I. The disposition of $[3 \mathrm{H}]$ norepinephrine, $[3 \mathrm{H}]$ dopamine and $[3 \mathrm{H}]$ dopa in various regions of the brain. $\mathrm{J}$ Neurochem 13:655-669

19. Folch J, Lees M, Sloane Stanley GH (1957) A simple method for the isolation and purification of total lipides from animal tissues. J Biol Chem 226:497-509

20. Seyer A, Boudah S, Broudin S, Junot C, Colsch B (2016) Annotation of the human cerebrospinal fluid lipidome using high resolution mass spectrometry and a dedicated data processing workflow. Metabolomics 12:91

21. Jensen DR, Schlaepfer IR, Morin CL et al (1997) Prevention of diet-induced obesity in transgenic mice overexpressing skeletal muscle lipoprotein lipase. Am J Phys 273:R683-R689

22. Eckel RH, Hernandez TL, Bell ML et al (2006) Carbohydrate balance predicts weight and fat gain in adults. Am J Clin Nutr 83:803808

23. Mullen TD, Hannun YA, Obeid LM (2012) Ceramide synthases at the centre of sphingolipid metabolism and biology. Biochem J 441: 789-802

24. Boslem E, MacIntosh G, Preston AM et al (2011) A lipidomic screen of palmitate-treated MIN6 $\beta$-cells links sphingolipid metabolites with endoplasmic reticulum (ER) stress and impaired protein trafficking. Biochem J 435:267-276

25. Wang H, Eckel RH (2012) Lipoprotein lipase in the brain and nervous system. Annu Rev Nutr 32:147-160

26. Bessesen DH, Richards CL, Etienne J, Goers JW, Eckel RH (1993) Spinal cord of the rat contains more lipoprotein lipase than other brain regions. J Lipid Res 34:229-238

27. Gray SJ, Foti SB, Schwartz JW et al (2011) Optimizing promoters for recombinant adeno-associated virus-mediated gene expression in the peripheral and central nervous system using selfcomplementary vectors. Hum Gene Ther 22:1143-1153

28. Miyashita Y, Shirai K, Itoh Y et al (2002) Low lipoprotein lipase mass in preheparin serum of type 2 diabetes mellitus patients and its recovery with insulin therapy. Diabetes Res Clin Pract 56:181-187

29. Frenais R, Nazih H, Ouguerram K et al (2001) In vivo evidence for the role of lipoprotein lipase activity in the regulation of apolipoprotein AI metabolism: a kinetic study in control subjects and patients with type II diabetes mellitus. J Clin Endocrinol Metab 86:1962-1967

30. Costabile G, Annuzzi G, Di Marino L et al (2011) Fasting and postprandial adipose tissue lipoprotein lipase and hormone-sensitive lipase in obesity and type 2 diabetes. J Endocrinol Investig 34: e110-e114

31. Annuzzi G, Giacco R, Patti L et al (2008) Postprandial chylomicrons and adipose tissue lipoprotein lipase are altered in type 2 diabetes independently of obesity and whole-body insulin resistance. Nutr Metab Cardiovasc Dis 18:531-538

32. Frangioudakis G, Diakanastasis B, Liao BQ et al (2013) Ceramide accumulation in L6 skeletal muscle cells due to increased activity of ceramide synthase isoforms has opposing effects on insulin action to those caused by palmitate treatment. Diabetologia 56:2697-2701

33. Benoit SC, Kemp CJ, Elias CF et al (2009) Palmitic acid mediates hypothalamic insulin resistance by altering PKC- $\theta$ subcellular localization in rodents. J Clin Invest 119:2577-2589 
34. Zhao L, Spassieva SD, Jucius TJ et al (2011) A deficiency of ceramide biosynthesis causes cerebellar purkinje cell neurodegeneration and lipofuscin accumulation. PLoS Genet 7:e1002063

35. Ginkel C, Hartmann D, vom Dorp K et al (2012) Ablation of neuronal ceramide synthase 1 in mice decreases ganglioside levels and expression of myelin-associated glycoprotein in oligodendrocytes. J Biol Chem 287:41888-41902
36. Contreras C, Gonzalez-Garcia I, Martinez-Sanchez N et al (2014) Central ceramide-induced hypothalamic lipotoxicity and ER stress regulate energy balance. Cell Rep 9:366-377

37. Cardinal P, Bellocchio L, Guzman-Quevedo O et al (2015) Cannabinoid type 1 (CB1) receptors on Sim1-expressing neurons regulate energy expenditure in male mice. Endocrinology 156:411-418 